ISSN 1997-5902

\title{
Détection biologique des résidus d'antibiotiques dans le lait et produits laitiers de vache consommés à Ouagadougou, Burkina Faso.
}

Touwendsida Serge Bagré*1, Serge Samandoulougou', Mahamady Traoré1, Daniel Illy¹, Gertrude BsadjoTchamba', Hadiza Bawa-lbrahim', S. Caroline Bouda' ${ }^{1}$, Alfred S. Traoré', Nicolas Barro'

${ }^{1}$ Laboratoire de Biologie Moléculaire, d'Epidémiologie et de Surveillance des Bactéries et Virus Transmissibles par les Aliments (LaBESTA), Centre de Recherches en Sciences Biologiques, Alimentaires et Nutritionnelles (CRSBAN), Ecole Doctorale Sciences et Technologies, 03 BP 7021 Ouagadougou 03, Université de Ouagadougou, Burkina Faso.

*Auteur pour correspondance : sergebagre@gmail.com; (+226) 70013717

Original submitted in on $15^{\text {th }}$ January 2015. Published online at www.m.elewa.org on 31st March 2015

http://dx.doi.org/10.4314/jab.v87i1.11

\section{RESUME}

Objectif : L'élevage traditionnel au Burkina n'associe pas généralement les bonnes pratiques vétérinaires. II se traduit par une utilisation anarchique des antibiotiques et le non-respect des délais d'attente. L'objectif est de mettre en évidence les résidus d'antibiotiques dans le lait et les produits laitiers consommés dans la ville de Ouagadougou, Burkina Faso.

Méthodologie et résultats : Au total, 201 échantillons de produits laitiers ont été collectés dans des marchés populaires et supermarchés puis analysés par la méthode biologique utilisant Bacillus subtilis ATCC 6633 et Geobacillus stearothermophilus ATCC 10149. Les aminosides et/ou quinolones et/ou macrolides et les bêta-lactamines et/ou sulfamides et/ou tétracyclines ont été détectés respectivement dans $17,24 \%$ et $51,72 \%$ des laits crus. Les aminosides et/ou quinolones et/ou macrolides ont été détectés dans $25 \%$ des laits caillés, 2,38\% des laits pasteurisés et 16,66\% des yaourts. Enfin, les bêta-lactamines et/ou sulfamides et/ou tétracyclines ont été détectée dans $70 \%$ des laits caillés, $66,66 \%$ des laits pasteurisés et $38,88 \%$ des yaourts.

Conclusion et application: Les laits et les produits laitiers consommés contiennent des résidus d'antibiotiques de différentes familles à des doses variées. Ainsi, des mesures pour la qualité des produits laitiers doivent être prises afin de préserver la santé des consommateurs.

Mots clés : Lait, Produits laitiers, Résidus d'antibiotiques, Bacillus subtilis, Geobacillus stearothermophilus.

Detection of antibiotics residues in dairy products sold in Ouagadougou, Burkina Faso.

ABSTRACT

Objective: The traditional breeding is generally associated with bad veterinary practices resulting in anarchical use of antibiotics. The objective of this study is to detect the antibiotic residues in milk and dairy products consumed in Ouagadougou, Burkina Faso.

Methods and Results: A total of 201 dairy products samples were collected in open markets and supermarkets then analyzed by the method using Bacillus subtilis ATCC 6633 and Geobacillus stearothermophilus ATCC 10149 for the detection of antibiotic residues in milk. Dairy products were tested 
positive for aminoglycosides and/or quinolones and/or macrolides (17.24\%) and beta-lactam and/or sulfonamides and/or tetracyclines (51.72\%). Residues of aminoglycosides and/or quinolones and/or macrolides were detected in $25 \%$ of curds, $2.38 \%$ of pasteurized milk and $16.66 \%$ of yoghurt while, the residues of beta-lactam and/or sulfonamides and/or tetracyclines were detected in $70 \%$ of curds, $66.66 \%$ of pasteurized milk and $38.88 \%$ of yoghurt.

Conclusion and Application: Milk and dairy products are contaminated by antibiotics residues of different families exceeding the authorized maximum limits. Therefore, measures to raise awareness of farmers and quality control of dairy products must be made to preserve the health of consumers.

Keywords: Milk, Dairy products, Antibiotic residues, Bacillus subtilis, Geobacillus stearothermophilus.

\section{INTRODUCTION}

La sécurité sanitaire des denrées alimentaires est une préoccupation de plus en plus importante en matière de santé publique (OMS, 2014). Les maladies d'origine alimentaire sont généralement causées par l'ingestion des agents pouvant être de nature chimique, physique ou infectieuse (bactéries, parasites, etc.) (OMS, 2007). Le lait, considéré comme un aliment complet occupe de plus en plus une place importante dans l'alimentation quotidienne des populations dans le monde en général et en Afrique subsaharienne en particulier. La production est essentiellement issue des vaches en élevage traditionnel; lors duquel les soins de santé des animaux sont faits de façon empirique impliquant une utilisation anarchique de produits pharmaceutiques dont les antibiotiques. Les antibiotiques sont largement utilisés dans les systèmes d'élevage en prophylaxie, ou comme additifs alimentaires ou facteurs de croissance des animaux (Zinedine et al., 2007 ; Ben-Madhi et Ouslimani, 2009). Ce type d'utilisation induit des changements sur la flore digestive des animaux entrainant l'émergence des souches résistantes (Fabre et al., 2000 ; Gysi, 2006). Aussi, le nonrespect des délais d'attente après les traitements entraine la présence des résidus d'antibiotiques dans les produits animaux dont le lait (Brouillet, 2002 ; Oliveira et al., 2006). L'émergence des microorganismes résistants aux antibiotiques est devenue une préoccupation majeure de l'organisation mondiale de la santé depuis l'apparition de souches pathogènes multirésistantes pour l'Homme causant des échecs thérapeutiques (Gedilaghine, 2005). En Afrique de l'Ouest, des bactéries telles que Staphylococcus aureus, Escherichia coli, Klebsiella pneumoniae, Streptococcus pneumoniae, Salmonella enterica, Shigella spp. d'origine clinique, environnementale et alimentaire se sont avérées de plus en plus résistantes aux antibiotiques couramment utilisés (Gassama et al., 2004 ; Bonkoungou et al., 2011 ; Bagré et al., 2014 ; Bsadjo-Tchamba et al., 2014). Dès lors, la recherche des causes des résistances des bactéries a porté sur le mode d'utilisation des antibiotiques dans l'élevage dans cette zone. Dans plusieurs pays d'Afrique, au regard des moyens techniques de détection, peu de données existent sur la présence des résidus d'antibiotiques dans les denrées alimentaires. En effet, des travaux ont mis en évidence dans des laits crus et pasteurisés, yaourts, fromages, crème fraîche, beurre et des boissons fermentées des résidus d'antibiotiques tels que les béta-lactamines, les macrolides, les pénicillines, les aminosides, les tétracyclines, le chloramphénicol à des doses importantes (Hilan et Chemali, 1998 ; Zinedine et al., 2007 ; Khaskheli et al., 2008 ; Ben-Madhi et Ouslimani, 2009 ; Mensah et al., 2014). Au Burkina Faso, il existe peu de données sur la présence des résidus d'antibiotiques dans les laits et produits laitiers. L'objectif de cette première étude est de mettre en évidence les résidus d'antibiotiques dans les produits laitiers ce qui indique une non observation des délais d'attente dans les élevages d'où l'existence de mauvaises pratiques vétérinaires au Burkina Faso. 


\section{Bagré et al. J. Appl. Biosci. Détection biologique des résidus d'antibiotiques dans le lait et produits}

laitiers de vache consommés à Ouagadougou, Burkina Faso.

\section{MATERIEL ET METHODES}

Type, période et site d'étude : II s'agit d'une étude prospective d'évaluation de la présence des résidus d'antibiotiques dans les produits laitiers de vaches. Les conditions d'élevage ont fait l'objet d'enquêtes. L'étude s'est déroulée d'avril à novembre 2013 dans neuf (9) villes du Burkina Faso (Figures 1a, 1b). La ville de Ouagadougou reçoit les laits des fermes situées dans les villages environnants.

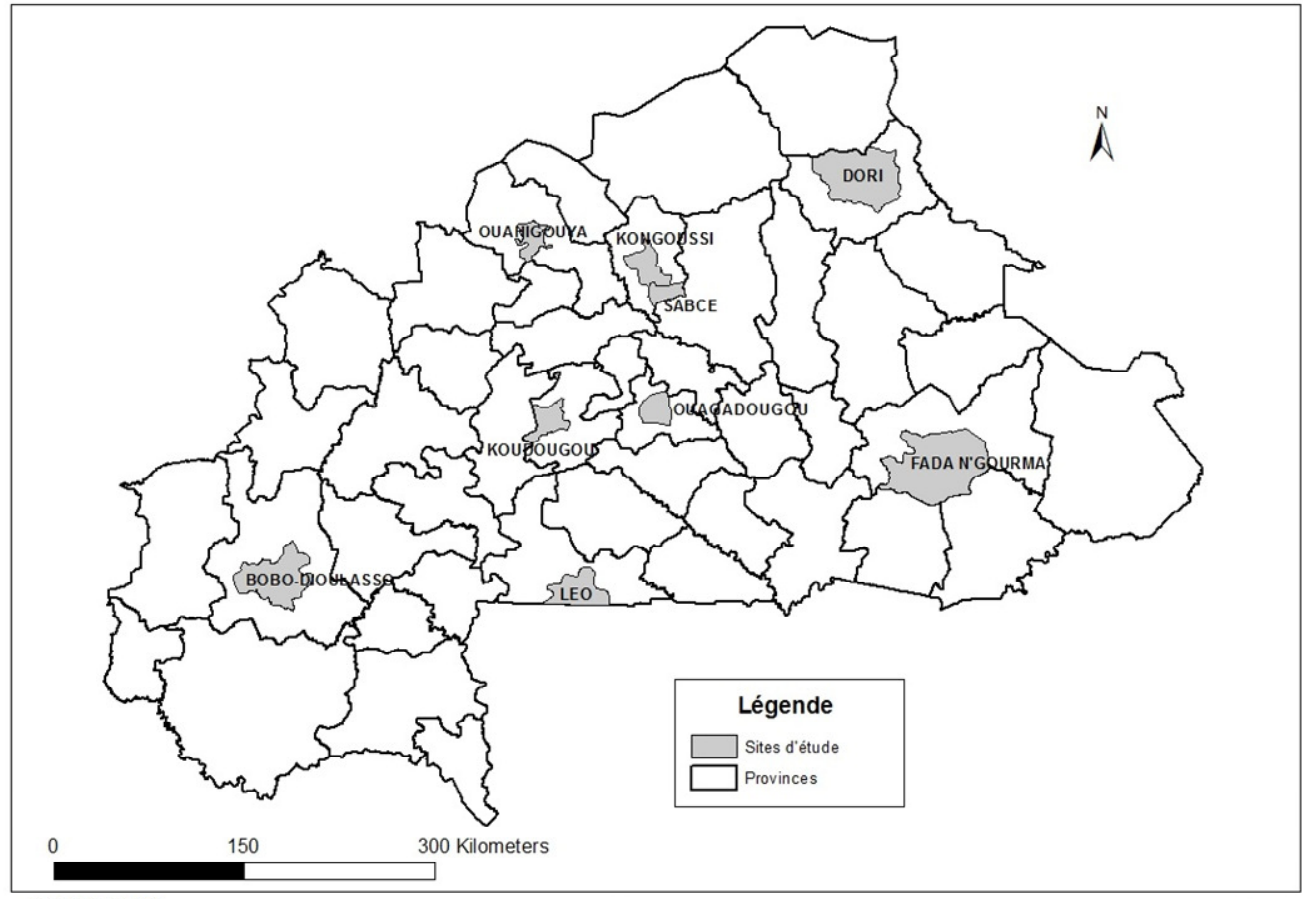

SOURCE : BNDT

(a)

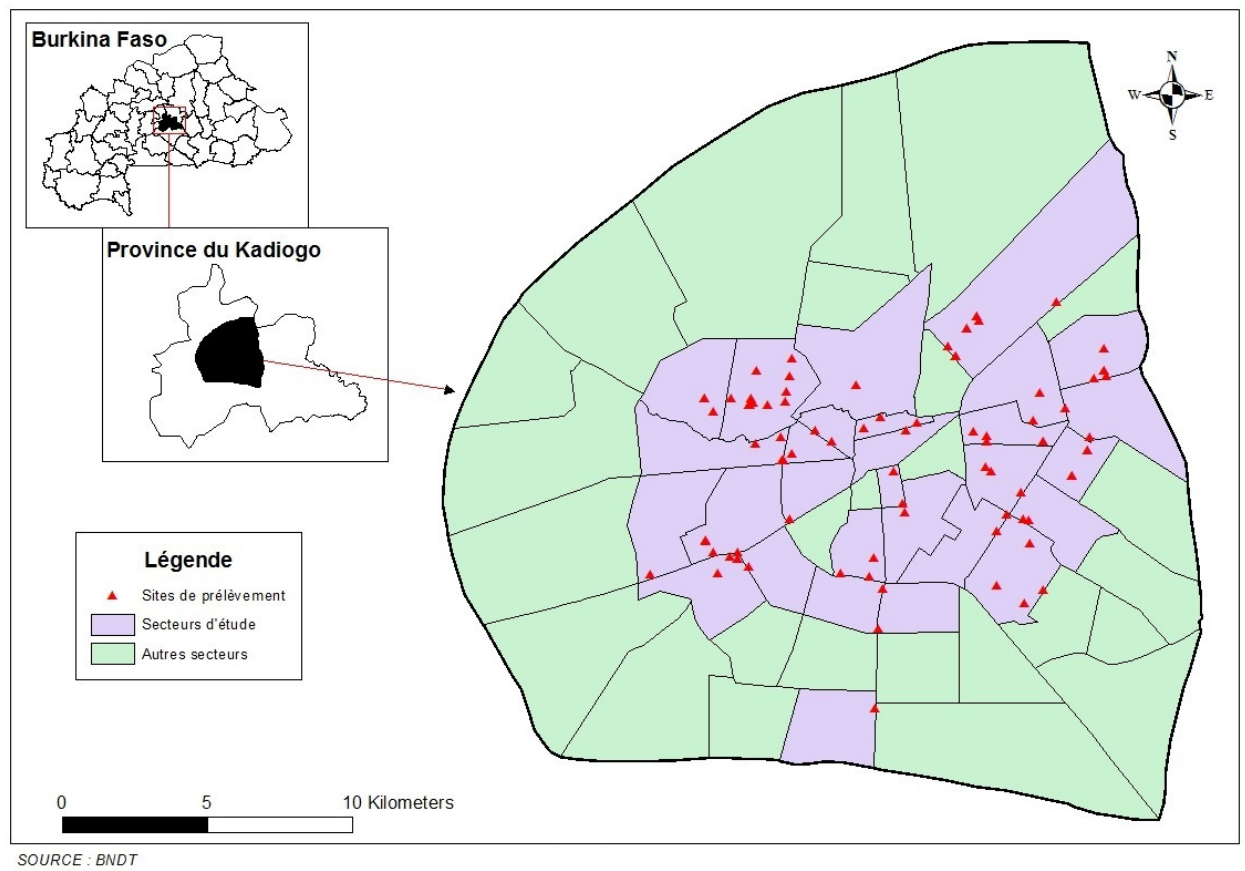

Figures 1 : (a) : Sites d'enquêtes ; (b) : Sites d'échantillonnage dans la ville de Ouagadougou, Burkina Faso. 


\section{Bagré et al. J. Appl. Biosci. Détection biologique des résidus d'antibiotiques dans le lait et produits}

laitiers de vache consommés à Ouagadougou, Burkina Faso.

Enquête: Des enquêteurs formés à cet effet ont questionnés les opérateurs de quarante un (41) élevages laitiers traditionnels, amélioré et moderne qui sont reliés à des unités de transformation de laits crus. Les questionnaires, selon le type de Millogo et al. (2008) ont été utilisés. Modifiés et adaptés à l'étude, ils incluaient les pratiques d'élevage et de traite, les soins vétérinaires ainsi que l'hygiène dans les unités d'élevage laitier. Les conditions de transport et la chaine de froid du lait cru issu ont été évaluées ainsi que l'identité des éleveurs. L'administration des questionnaires a été faite par interview direct des éleveurs (41) et en observant les pratiques d'hygiène au sein des fermes.

Échantillonnage: L'échantillonnage a concerné les produits laitiers de production traditionnelle, sémiindustrielle et industrielle. Les échantillons de laits crus $(n=29)$, laits caillés ( $n=40)$, laits pasteurisés $(n=42)$ et yaourt $(n=90)$ ont été achetés dans les marchés populaires et les supermarchés. Les échantillons de 125 à $500 \mathrm{ml}$ de volume ont été acheminés au laboratoire dans une glacière à moins de $15^{\circ} \mathrm{C}$ pour être traités et analysés. Une quantité de $5 \mathrm{ml}$ a été aliquotée puis conservée au congélateur à $-20^{\circ} \mathrm{C}$ pour les analyses.

Détection des résidus d'antibiotiques : La présence des résidus d'antibiotiques a été détectée dans les différents échantillons de produits laitiers selon la méthode décrite par Ben-Mahdi et Ouslimani (2009) basée sur la réaction d'inhibition de la croissance des bactéries tests. Bacillus subtilis est sensible aux antibiotiques de la famille des aminosides, des quinolones et des macrolides alors que Geobacillus stearothermophilus est sensible aux béta-lactamines, aux sulfamides et aux tétracyclines. Pour ce faire, les

\section{RESULTATS}

Caractéristiques des unités d'élevage: Les enquêtes ont révélés l'existence de deux (02) types d'élevage (Tableau 1): le type traditionnel et le type amélioré. Ils se distinguent par la conduite des pratiques et l'organisation du système d'élevage. Les opérations de traite sont manuelles dans les deux types. La majorité des unités d'élevage sont de type traditionnel $(75,61 \%)$. L'ethnie «Peulh » $(95,12 \%)$ pratique principalement l'élevage des bovins contre une souches de références de Geobacillus stearothermophilus ATCC 10149 et de Bacillus subtilus ATCC 6633 ont été enrichies chacune dans un bouillon Mueller Hinton (MH) (Liofilchem, Italie) et incubées respectivement à $55^{\circ} \mathrm{C}$ et $30^{\circ} \mathrm{C}$. Après $24 \mathrm{~h}$ d'incubation, une quantité de $0,1 \mathrm{ml}$ des suspensions de Geobacillus stearothermophilus et Bacillus subtilis a été ensemencée sur gélose $\mathrm{MH}$ puis incubée à la température appropriée afin d'avoir des colonies pures bien distinctes. Les suspensions des souches ont été préparées en homogénéisant les colonies pures dans de l'eau physiologique ( $\mathrm{NaCl} 9 \mathrm{~g} / \mathrm{L}$ d'eau) puis ajusté à la densité optique (DO) 0,08-0,1 par évaluation au spectrophotomètre à $625 \mathrm{~nm}$ (équivalent au standard McFarland 0.5). Les boîtes ont été ensuite inondées par ces suspensions bactériennes. Les échantillons de lait cru, lait caillé, lait pasteurisé et de yaourt ont été d'abord chauffés à $80^{\circ} \mathrm{C}$ pendant 5 à 10 minutes afin d'inactiver la lyzozyme et de détruire d'éventuels germes. Ils ont été ensuite centrifugés à $1000 \mathrm{~g}$ pendant $1 \mathrm{mn}$ à la température de $25^{\circ} \mathrm{C}$. Des disques de 6,13 $\mathrm{mm}$ de diamètre stériles ont été imprégnés des surnageants obtenus de produits laitiers puis déposés sur la gélose $\mathrm{MH}$. Les boîtes ont été incubées respectivement à $55^{\circ} \mathrm{C}$ et $30^{\circ} \mathrm{C}$ pour Geobacillus stearothermophilus et Bacillus subtilis. Après $24 \mathrm{~h}$ d'incubation, les zones claires autour des disques de chaque échantillon positif ont été mesurées à l'aide d'un pied à coulisse électronique (STAINLESS HARDENED).

Analyses des données: Les données des enquêtes ont été saisies et analysées avec Epi Info version 7 et Microsoft ${ }^{8}$ Excel 2010. Les fréquences ont été calculées avec un intervalle de confiance (IC) de 95\% et une marge d'erreur de $5 \%(0,05)$.

faible implication des autres ethnies $(4,88 \%)$. La traite dans les unités est assurée par les femmes $(80,49 \%)$ qui sont pour la plupart illettrée et ignorantes des bonnes pratiques d'hygiènes $(97,56 \%)$. Pendant l'opération de traite, il y'a la présence d'insectes (mouches, fourmis), dans un environnement traditionnel de type rural. Pendant la distribution, le lait cru collecté n'est pas réfrigéré. 
Tableau 1 : Caractéristiques des unités d'élevage au Burkina Faso.

\begin{tabular}{|c|c|c|}
\hline \multirow[t]{2}{*}{ Paramètres } & \multicolumn{2}{|c|}{ Type d'élevage } \\
\hline & Amélioré ( $n=10)$ & Traditionnel $(n=31)$ \\
\hline \multicolumn{3}{|l|}{ Formation en santé animale } \\
\hline Oui & $9(21,95 \%)$ & $6(14,63 \%)$ \\
\hline Non & $1(2,44 \%)$ & $25(60,98 \%)$ \\
\hline \multicolumn{3}{|c|}{ Supplémentation des aliments avec des antibiotiques } \\
\hline Oui & $10(24,39 \%)$ & $15(36,56 \%)$ \\
\hline Non & 0 & $16(39,02 \%)$ \\
\hline \multicolumn{3}{|l|}{ Fréquences de nettoyage des fèces } \\
\hline$\leq 3$ fois / jour & $8(19,51 \%)$ & $18(43,90 \%)$ \\
\hline$\leq 3$ fois / semaine & $2(4,88 \%)$ & $3(7,32 \%)$ \\
\hline Jamais & $0(0 \%)$ & $10(24,39 \%)$ \\
\hline \multicolumn{3}{|l|}{ Désinfectants utilisés lors de la traite } \\
\hline Savon & $10(24,39 \%)$ & $30(73,17 \%)$ \\
\hline Autre (détergents) & $0(0 \%)$ & $1(2,44 \%)$ \\
\hline \multicolumn{3}{|l|}{ Nettoyage du pis avant la traite } \\
\hline Oui & $7(17,07 \%)$ & $13(31,71 \%)$ \\
\hline Non & $3(7,32 \%)$ & $18(43,90 \%)$ \\
\hline \multicolumn{3}{|l|}{ Ustensiles de traite utilisés } \\
\hline Calebasse & $8(19,51 \%)$ & $22(53,66 \%)$ \\
\hline Ustensile en plastique & $2(4,88 \%)$ & $9(21,95 \%)$ \\
\hline \multicolumn{3}{|l|}{ Nettoyage des ustensiles avant la traite } \\
\hline Oui & $10(24,39 \%)$ & $27(65,85 \%)$ \\
\hline Non & $0(0 \%)$ & $4(9,76 \%)$ \\
\hline \multicolumn{3}{|l|}{ Ustensiles de transport du lait } \\
\hline Bidon en plastique & $10(24,39 \%)$ & $30(73,17 \%)$ \\
\hline Ustensile en aluminium & $0(0 \%)$ & $1(2,44 \%)$ \\
\hline \multicolumn{3}{|l|}{$\begin{array}{l}\text { Mode de transport du lait collecté vers les unités } \\
\text { de transformations }\end{array}$} \\
\hline Motocyclette & $1(2,44 \%)$ & $9(21,95 \%)$ \\
\hline Pied & $5(12,19 \%)$ & $12(29,27 \%)$ \\
\hline Vélo & $4(9,76 \%)$ & $10(24,39 \%)$ \\
\hline
\end{tabular}

Taux de contamination des produits laitiers: Les résultats ont montré que $59,70 \%$ des produits laitiers consommés à Ouagadougou contenaient des résidus d'antibiotiques. II ressort également de cette étude que $70 \%$ et $25 \%$ des laits caillés; $66,66 \%$ et $2,38 \%$ des laits pasteurisés ; $51,72 \%$ et $17,24 \%$ des laits crus et $38,88 \%$ et $16,66 \%$ des yaourts renfermaient des résidus de beta-lactamines et/ou sulfamides et/ou tétracyclines et des résidus d'aminosides et/ou quinolones et/ou macrolides (Tableau 2).

Tableau 2 : Proportion des produits laitiers testés positifs aux résidus d'antibiotiques.

\begin{tabular}{lccc}
\hline Types de lait & \multicolumn{3}{c}{ Échantillons positifs } \\
\cline { 2 - 4 } & Bacillus subtilis & $\begin{array}{c}\text { Geobacillus } \\
\text { stearothermophilus }\end{array}$ & $\begin{array}{c}\text { Bacillus subtilis et Geobacillus } \\
\text { stearothermophilus }\end{array}$ \\
\cline { 2 - 4 } Lait cru $(n=29)$ & $5(17,24 \%)[7-10 \mathrm{~mm}]$ & $15(51,72 \%)[7-15 \mathrm{~mm}]$ & $4(13,79 \%)$ \\
Lait caillé $(n=40)$ & $10(25 \%)[7-12 \mathrm{~mm}]$ & $28(70 \%)[7-16 \mathrm{~mm}]$ & $8(20 \%)$ \\
Lait pasteurisé $(\mathrm{n}=42)$ & $1(2,38 \%)[7 \mathrm{~mm}]$ & $28(66,66 \%)[8-16 \mathrm{~mm}]$ & $1(2,38 \%)$ \\
Yaourt $(\mathrm{n}=90)$ & $15(16,66 \%)[7-10 \mathrm{~mm}]$ & $35(38,88 \%)[7-13 \mathrm{~mm}]$ & $4(4,44 \%)$ \\
\hline Total $(\mathrm{n}=201)$ & $31(15,42 \%)$ & $106(52,74 \%)$ & $17(8,46 \%)$ \\
\hline $\mathrm{n}=$ nombre d'échantillons par type de lait ; ()$=$ Pourcentage de positif ; [ ] = Diamètres d'inhibitions minimal et maximal mesurés.
\end{tabular}




\section{Bagré et al. J. Appl. Biosci. Détection biologique des résidus d'antibiotiques dans le lait et produits}

laitiers de vache consommés à Ouagadougou, Burkina Faso.

L'inhibition annulaire des souches de références a montrée des diamètres plus élevés avec Geobacillus stearothermophilus qu'avec Bacillus subtilis quel que

\section{DISCUSSION}

Les résultats de l'enquête montrent des caractéristiques variées des types d'élevage des bovins au Burkina. II n'existe pas un élevage exclusif de vache laitière. L'élevage traditionnel de type transhumant ou sédentaire conduit par l'ethnie Peulh est le système dominant (Hamadou et Sanon, 2005). Ce mode d'élevage au Burkina Faso est dominé par des pratiques traditionnelles caractérisées par la traite manuelle et l'utilisation des soins vétérinaires empiriques. Des études ont montré que dans les pays développés, les antibiotiques sont utilisés dans les fermes bovines laitières principalement pour le traitement des mammites (37\%) et des problèmes de locomotion (14\%) (Cazeau et al., 2010). Ces pathologies sont fréquentes sur les bovins au Burkina (Traoré et al., 2004). En effet, l'environnement d'élevage n'est pas favorable à la santé des animaux d'où les problèmes sanitaires des bêtes entrainant l'utilisation de produits de soins vétérinaires notamment les antibiotiques. Cependant, dans certains pays, il est possible de se procurer des antibiotiques sans ordonnance. Ces derniers sont très utilisés chez les animaux. Ces deux aspects peuvent expliquer l'augmentation de la résistance aux antibiotiques. Une proportion des produits laitiers consommés à Ouagadougou renferment des résidus d'antibiotiques appartenant aux familles de bêta-lactamines, sulfamides, tétracyclines et dans une moindre mesure aux familles d'aminosides, de macrolides et de quinolones à des taux variés. Toutes ces molécules, très utilisées en médecine humaine le sont aussi en élevage au Burkina Faso. Les travaux de plusieurs auteurs en Afrique, Asie et Amérique latine ont mis en évidence les résidus de cefquinome de la famille des céphalosporines, de bêta-lactamines, de tétracyclines, d'oxytétracyclines, de chloramphénicol et de sulfamides dans les laits (Hilan et Chemali, 1998; Arora et Chhabra, 2004 ; Zinedine et al., 2007 ; Khaskheli et al., 2008 ; Ben-Mahdi et Ouslimani, 2009 ; Mensah et al., 2014 ; Randall et al., 2014 ; Redding et al., 2014). En effet, ces antibiotiques sont largement utilisés en médecine vétérinaire pour les soins des vaches

\section{CONCLUSION}

II ressort de cette étude menée au Burkina Faso que les produits laitiers consommés dans la ville de soit le produit laitier. De grandes proportions de produits laitiers ont montré des activités inhibitrices sur les deux souches de bactéries.

laitières (Schwarz et Chaslus-Dancla, 2001 ; Chevance et Moulin, 2007; Cazeau et al., 2010). Au Burkina Faso, la brucellose, la tuberculose et les mammites bovines sont les principales pathologies qui affectent l'élevage bovin (Traoré et al., 2004) entrainant une forte demande d'antibiotiques à l'importation (MRA, 2009). Ainsi, ces antibiotiques sont utilisés à d'autres fins c'est-à-dire comme facteurs de croissance ou comme additifs alimentaires selon l'enquête effectuée auprès des fermes laitières. Ce phénomène a été signalé par plusieurs auteurs (Zinedine et al., 2007 ; Khaskheli et al., 2008; Ben-Madhi et Ouslimani, 2009). Ces antibiotiques administrés souvent par voie intramammaire passent dans le lait de traite à des concentrations élevées (Jepsen, 1998). En effet, au Burkina Faso, il n'existe aucune règlementation régissant le contrôle systématique des laits crus avant la transformation. Les travaux de Randall et al. (2014) ont montré qu'il existe une corrélation entre la présence de résidus d'antibiotiques et l'émergence de Escherichia coli résistant aux familles des céphalosporines. La présence des résidus d'antibiotiques dans les aliments présente aussi des risques d'émergence de bactéries résistantes (Gysi, 2006). En effet, l'ingestion de ces petites doses d'antibiotiques dans le lait conduit au développement de bactéries résistantes. Des travaux ont montré que les produits laitiers sont contaminés à divers degré par des E. coli notamment ECEP, ECEH 0157, Salmonella spp., Staphylococcus aureus, Enterococcus spp. (Bonfoh et al., 2002 ; Solomakos et al., 2009 ; Aggad et al., 2010; Dadie et al., 2010; Savadogo et al., 2010; Katinan et al., 2012; Bagré et al., 2014). D'autres travaux sur certaines souches de $E$. coli et Salmonella spp. isolées des produits laitiers en Grèce, au Burkina Faso et dans d'autres pays ont montré des résistances vis-à-vis de l'amoxicilline, de l'amoxicilline-acide clavulanique, du triméthroprime sulfamethoxazole, de l'érythromycine, de la tobramycine, du chloramphénicol et des sulfamides (Solomakos et al., 2009; Savadogo et al., 2010 ; Bagré et al., 2014).

Ouagadougou contiennent à divers degrés des résidus d'antibiotiques. L'exposition des agents bactériens des 


\section{Bagré et al. J. Appl. Biosci. Détection biologique des résidus d'antibiotiques dans le lait et produits}

laitiers de vache consommés à Ouagadougou, Burkina Faso.

animaux (vaches) à ces résidus d'antibiotiques peuvent être à l'origine de l'émergence de souches résistantes ou multirésistantes. Au regard des risques pour la santé humaine, des mesures et des réglementations

\section{REMERCIEMENTS}

Ce travail a été effectué grâce au soutien de la Fondation Internationale pour la Science (IFS) pour son allocation E/5558. Les auteurs remercient également Dr Jules André Ilboudo et Dr Aly Savadogo du Centre de Recherches en Sciences Biologiques, Alimentaires

\section{REFERENCES BIBLIOGRAPHIQUES}

Aggad $H$, Bridja $M$, Bouhai $A$, Benaouali M, Djebli $A$, 2010. Some quality aspects of pasteurized milk in Algeria. World Journal of Dairy and Food Science 5: 21-24.

Arora A, Chhabra D, 2004. Screening for antimicrobial residues in milk by disc assay. Indian Veterinary Journal 81: 1400-1401.

Bagré TS, Kagambèga $A$, Bawa $\mathrm{IH}$, Bsadjo Tchamba G, Dembélé R, Zongo C, Savadogo A, Aggad $\mathrm{H}$, Traoré AS, Barro N, 2014. Antibiotic susceptibility of Escherichia coli and Salmonella strains isolated from raw and curds milk consumed in Ouagadougou and Ziniaré, Burkina Faso. African Journal of Microbiology Research 8: 1012-1016.

Ben-Mahdi MH, Ouslimani S, 2009. Mise en évidence de résidus d'antibiotiques dans le lait de vache produit dans l'Algérois. European Journal of Scientific Research 36: 357-362.

Bonfoh B, Fané A, Traoré NA, Coulibaly Z, Simbé CF, Alfaroukh OI, Nicolet J, Farah Z, Zinsstag J, 2002. Qualité microbiologique du lait et des produits laitiers vendus en saison chaude dans le district de Bamako au Mali. BIOTERRE, Revue Internationale de la Science de la Vie et de la Terre. $N^{\circ}$ spécial. 242-250.

Bonkoungou IJO, Lienemann T, Martikainen $\mathrm{O}$, Dembele R, Sanou I, Traore AS, Sutonen A, Barro N, Haukka K, 2011. Diarrhoeagenic Escherichia coli detected by 16 plex PCR in children with and without diarrhea in Burkina Faso. Clinical Microbiology and Infection 18: 901-906.

Brouillet $P, 2002$. Résidus de médicaments dans le lait et tests de détection. Bulletin des GVT 15: 2541. doivent être prises par les scientifiques, les autorités compétentes, les éleveurs et les industriels laitiers pour le contrôle systématique des laits crus et des produits laitiers afin d'éviter les différents risques y afférents.

et Nutritionnelles (CRSBAN)/ Université de Ouagadougou respectivement pour Geobacillus stearothermophilus ATCC 10149 et Bacillus subtilis ATCC 6633.

Bsadjo-Tchamba G, Bawa IH, Nzouankeu A, Bagré TS, Dembélé R, Bonkoungou IJO, Zongo C, Savadogo A, Traoré AS, Barro N, 2014. Occurrence and antimicrobial susceptibility of Escherichia coli and Salmonella spp. isolated from "zoom-koom" beverage and ice in Ouagadougou, Burkina Faso. African Journal of Microbiology Research 8: 3243-3249.

Cazeau G, Chazel M, Jarrige N, Sala C, Calavas D, Gay E, 2010. Utilisation des antibiotiques par les éleveurs en filière bovine en France. Rencontres autour de la Recherche sur les Ruminants 17: 71-74.

Chevance A, Moulin G, 2007. Suivi des ventes de médicaments vétérinaires contenant des antibiotiques en France en 2006. Fougères, France, AFSSA-ANMV $38 \mathrm{p}$.

Dadie A, Nzebo D, Guessennd N, Dako E, Dosso M, 2010. Prévalence de Escherichia coli entéropathogènes dans le lait non pasteurisé produit à Abidjan, Côte d'Ivoire. International Journal of Biological and Chemical Sciences 4: 11-18.

Fabre JM, Gardey L, Lherbette L, De Boisseson M, Berthelot X, 2000. Détection des résidus de céfalexine dans le lait en cas d'allongement de la durée du traitement par voie intramammaire. Revue de Médecine Vétérinaire 151: 965-968.

Gassama A, Aidara-Kane A, Chainier D, Denis F, Ploy MC, 2004. Integron-associated antibioticresistance in enteroaggregative and enteroinvasive Escherichia coli. Microbial Drug Resistance 10: 27-30

Gedilaghine V, 2005. La rationalisation du traitement des mammites en exploitation laitière: Conception et réalisation d'une enquête d'évaluation de la mise en place de l'action GTV partenaire dans le département de la 
manche. Thèse. École Nationale Vétérinaire d'Alfort ; France 106p.

Gysi M, 2006. Antibiotiques utilisés en production laitière en 2003 et 2004. Revue Suisse Agriculture 38: 215-220.

Hamadou S, Sanon Y, 2005. Synthèse bibliographique sur les filières laitières au Burkina Faso. Repol, Bobo-Dioulasso, Burkina Faso. CIRDES/MRA (Documents de travail $n^{\circ} 3$ ). $48 p$.

Hilan C, Chemali Z, 1998. La contamination des produits laitiers par les antibiotiques au Liban. Annales de recherche scientifique 1: 267-275.

Jepsen A, 1998. Les résidus de désinfectants et d'antibiotiques dans le lait. WHO_MONO 48: 457-464

Katinan CR, Sadat AW, Chatigre KO, Bohoussou KM, Assidjo NE, 2012. Évaluation de la qualité des laits cailles artisanaux produits et consommés dans Yamoussoukro. Journal of Applied Biosciences 55: 4020-4027.

Khaskheli M, Malik RS, Arain MA, Soomro AH, Arain $\mathrm{HH}, 2008$. Detection of beta-lactam antibiotic residues in market milk. Pakistan Journal of Nutrition 7: 682-685.

Mensah SEP, Aboh AB, Salifou S, Mensah GA, Sanders P, Abiola FA, Koudandé OD, 2014. Risques dus aux résidus d'antibiotiques détectés dans le lait de vache produit dans le Centre Bénin. Journal of Applied Biosciences 80: 7102-7112.

Millogo V, Ouédraogo GA, Agenäs S, SvennerstenSjannja K, 2008. Survey on dairy cattle milk production and milk quality problems in periurban areas in Burkina Faso. African Journal of Agricultural Research 3: 215-224.

Ministère des ressources animales (MRA), 2009. Enquête Nationale sur les Effectifs du Cheptel. Annuaire statistique du secteur de l'élevage. Direction des statistiques animales du Burkina Faso.

Oliveira R, De Pietro A, Cass Q, 2006. Quantification of cephalexin as residue levels in bovine milk by high-performance liquid chromatography with on-line sample cleanup. Talanta 71: 12331238.

Oliver SP, Jayarao BM, Almeida RA, 2005. Foodborne pathogens in milk and the dairy farm environment: Food safety and public health implications. Foodborne Pathogens and Disease 2: 115-129.
Organisation Mondiale de la Santé (OMS), 2007. Salubrité des aliments et maladies d'origine alimentaire. Aide mémoire $\mathrm{n}^{\circ} 237$.

Organisation mondiale de la santé (OMS), 2014. Antimicrobial Resistance: Global Report on Surveillance 2014. OMS 257p.

Randall L, Heinrich K, Horton R, Brunton L, Sharman M, Bailey-Horne V, Sharma M, McLaren I, Coldham N, Teale C, Jones J, 2014. Detection of antibiotic residues and association of cefquinome residues with the occurrence of Extended-Spectrum $\beta$-Lactamase (ESBL)producing bacteria in waste milk samples from dairy farms in England and Wales in 2011. Research in Veterinary Science 96:15-24.

Redding LE, Cubas-Delgado F, Sammel MD, Smith G, Galligan DT, Levy MZ, Hennessy S, 2014. Antibiotic residues in milk from small dairy farms in rural Peru. Food Additives and Contaminants 31:1001-1008.

Savadogo A, Ouattara CAT, Ilboudo AJ, Karou K, Traoré AS, 2010. Isolation and susceptibilty to antibiotics of bacterial strains from Burkina Faso fermented milk samples. Advance Journal of Food Science and Technology 2: 91-95.

Schwarz S, Chaslus-Dancla E, 2001. Use of antimicrobials in veterinary medicine and mechanisms of resistance. Veterinary Research 32: 201-225.

Solomakos N, Govaris A, Angelidis AS, Pournaras S, Burriel AR, Kritas KS, Papageorgiou DK, 2009. Occurrence, virulence genes and antibiotic resistance of Escherichia coli 0157 isolated from raw bovine, caprine and ovine milk in Greece. Food Microbiology 26: 865-871.

Traoré A, Tamboura HH, Bayala B, Rouamba DW, Yaméogo N, Sanou M, 2004. Prévalence globale des pathologies majeures liées à la production laitière bovine en système d'élevage intra-urbain à Hamdallaye (Ouagadougou). Biotechnologie, Agronomie, Société et Environnement 8: 3-8.

Zinedine A, Faid M, Benlemlih M, 2007. Détection des résidus d'antibiotiques dans le lait et les produits laitiers par méthode microbiologique. Revue de Microbiologie Industrielle, Sanitaire et Environnementale 1: 1-9. 